\title{
Effect of Parasympathetic Stimulation on Brain Activity During Appraisal of Fearful Expressions
}

\author{
Elena Makovac*,', Sarah N Garfinkel ${ }^{2,3}$, Andrea Bassi', Barbara Basile', Emiliano Macaluso', \\ Mara Cercignani ${ }^{1,5}$, Giovanni Calcagnini ${ }^{6}$, Eugenio Mattei ${ }^{6}$, Daniela Agalliu ${ }^{4}$, Pietro Cortelli ${ }^{7,8}$, \\ Carlo Caltagirone ${ }^{4,9}$, Marco Bozzali' and Hugo Critchley ${ }^{2,3}$ \\ 'Neuroimaging Laboratory, IRCCS Santa Lucia Foundation, Rome, Italy; ${ }^{2}$ Psychiatry, Brighton and Sussex Medical School, University of Sussex, \\ Falmer, Brighton, UK; ${ }^{3}$ Sackler Centre for Consciousness Science, University of Sussex, Falmer, Brighton, UK; ${ }^{4}$ Department of Clinical and \\ Behavioural Neurology, IRCCS Santa Lucia Foundation, Rome, Italy; ${ }^{5}$ Brighton \& Sussex Medical School, Clinical Imaging Sciences Centre, \\ University of Sussex, Brighton, UK; ${ }^{6}$ Department of Technology and Health, Italian Institute of Health, Rome, Italy; ${ }^{7}$ Neurological Clinic, DIBIDEM, \\ Alma Mater Studiorum-University of Bologna, Bologna, Italy; ${ }^{8}$ RCCS Institute of Neurological Sciences of Bologna, Bologna, Italy; ${ }^{9}$ Department of \\ Neuroscience, University of Rome 'Tor Vergata', Rome, Italy
}

\begin{abstract}
Autonomic nervous system activity is an important component of human emotion. Mental processes influence bodily physiology, which in turn feeds back to influence thoughts and feelings. Afferent cardiovascular signals from arterial baroreceptors in the carotid sinuses are processed within the brain and contribute to this two-way communication with the body. These carotid baroreceptors can be stimulated non-invasively by externally applying focal negative pressure bilaterally to the neck. In an experiment combining functional neuroimaging ( $\mathrm{FMRI}$ ) with carotid stimulation in healthy participants, we tested the hypothesis that manipulating afferent cardiovascular signals alters the central processing of emotional information (fearful and neutral facial expressions). Carotid stimulation, compared with sham stimulation, broadly attenuated activity across cortical and brainstem regions. Modulation of emotional processing was apparent as a significant expression-by-stimulation interaction within left amygdala, where responses during appraisal of fearful faces were selectively reduced by carotid stimulation. Moreover, activity reductions within insula, amygdala, and hippocampus correlated with the degree of stimulationevoked change in the explicit emotional ratings of fearful faces. Across participants, individual differences in autonomic state (heart rate variability, a proxy measure of autonomic balance toward parasympathetic activity) predicted the extent to which carotid stimulation influenced neural (amygdala) responses during appraisal and subjective rating of fearful faces. Together our results provide mechanistic insight into the visceral component of emotion by identifying the neural substrates mediating cardiovascular influences on the processing of fear signals, potentially implicating central baroreflex mechanisms for anxiolytic treatment targets.

Neuropsychopharmacology (20I5) 40, 1649-1658; doi:I0.I038/npp.20I5. I0; published online 4 March 2015
\end{abstract}

\section{INTRODUCTION}

Influential theories emphasize the contribution of bodily arousal to emotion and affective feelings (James, 1894; Damasio, 1994; Barrett et al, 2007; Dolan, 2002). Even without a specific mapping of bodily state to emotional type (eg Cannon, 1927), the cognitive interpretation of a change in bodily arousal can shape the emotional experience (Schachter and Singer, 1962; Barrett, Lindquist and Gendron, 2007). Improved anatomical and functional description of bidirectional interactions between body and brain has advanced our understanding of emotional mechanisms (Craig, 2002; Critchley et al, 2002, 2004, Critchley, 2005a, b; Harrison et al, 2009) and, for some emotions, there is good evidence of specific coupling with

*Correspondence: Dr E Makovac, Neuroimaging Laboratory, Santa Lucia Foundation, Via Ardeatina 306, Rome, Italy, Tel: +39 065150 I324, Fax: +39065150 1213, E-mail: e.makovac@hsantalucia.it

Received 17 July 2014; revised I December 2014; accepted 29 December 2014; accepted article preview online 12 January 2015 autonomically mediated changes in peripheral physiology (Ekman et al, 1983; Harrison et al, 2006).

There is particular interest in the relationship between parasympathetic control of the heart and emotion. Here, heart rate variability (HRV) (derived from electrocardiography) indexes the parasympathetic regulation of the heart via the vagus nerve and reflects the degree to which the cardiac activity can be modulated to meet changing situational and emotional demands (Thayer and Lane, 2000; Thayer and Brosschot, 2005). Moreover, it is closely linked to individual differences in emotional responding, and capacity for self-regulation (see Friedman, 2007; Thayer et al, 2009). Increased HRV is associated with adaptive emotional responses to threat (Thayer and Lane, 2000; Thayer et al, 2009) and increased sensitivity to the emotions of others (Quintana et al, 2012).

The human amygdala mediates interaction between the body and the brain during affective processing. The amygdala supports the perception of fear signals and threat (Zald, 2003; Phelps and LeDoux, 2005), and its activity correlates with the emotional intensity rating of affective 
pictures (Phan et al, 2004), including facial expressions (Hamann and Mao, 2002). Outputs from the amygdala innervate hypothalamic and brainstem autonomic circuits to trigger autonomic arousal responses to emotional challenges, particularly threats (LeDoux, 2000). Amygdalainduced autonomic arousal is expressed as increased sympathetic activity and/or decreased HRV (Critchley et al, 2005b; Gianaros et al, 2012). The amygdala is also sensitive to feedback from the periphery regarding state of bodily arousal (Critchley et al, 2002).

Carotid baroreceptors signal the state of cardiovascular arousal to the brain: when arterial blood pressure rises, baroreceptors drive afferent neuronal firing to evoke a reflexmediated increase in parasympathetic activity and a decrease in sympathetic activity (Sagawa, 1983; reviewed in Fadel et al, 2003). These natural fluctuations in baroreceptor activity (with cardiac cycle) can influence different cognitive functions. Cardiac cycle has modulatory effects on simple reaction time in different sensory modalities (Edwards et al, 2008), somatosensory thresholds (Wilkinson et al, 2013), on memory for words (Garfinkel et al, 2013), and processing of fear (Garfinkel et al, 2014). Although the literature argues for an inhibitory effect of systole, recent data (Garfinkel et al, 2014) show an enhancing effect on fear processing, and others report reduction of somatosensory thresholds (eg, Edwards et al, 2008; Wilkinson et al, 2013). Speculatively, these discrepancies suggest two competing mechanisms that may reflect different roles and afferent channels (cranial nerves IX and $\mathrm{X}$, respectively) of carotid and cardiac baroreceptors. In fact, diastolic activation of low pressure cardiac baroreceptors during cardiac filling is known to inhibit neurons within the locus coeruleus (Morilak et al, 1986; Jacobs et al, 1991).

Artificial mechanical stimulation of baroreceptor activity also demonstrates interaction between autonomic and cognitive/emotional processes (Calcagnini et al, 2010; Basile et al, 2013a,b). A non-invasive, automated neck suction device enables carotid stimulation (CS) to be implemented concurrently with functional magnetic resonance neuroimaging (fMRI): neck suction stimulates carotid baroreceptors by increasing transmural pressure within the carotid sinus (Cooper and Hainsworth; 2009). This evokes an enhanced parasympathetic cardiovascular drive via the baroreflex. Previous studies from our group show that the same CS procedure modulates activity within brain areas including amygdala and insula in brain at rest, when engaged in a cognitive task, or during emotional processing (Basile et al, 2013a,b).

Motivated by recent observations regarding the influence of cardiac cycle on fear processing (Garfinkel et al, 2014), the aim of the present study was to investigate the effect of a direct parasympathetic perturbation by CS on neural and subjective responses to the appraisal of fearful (compared with neutral) facial expressions. Further, we test whether individual traits in basal vagal tone (expressed as resting HRV) predict the degree to which this perturbation of viscerosensory autonomic function influences fear processing. On the basis of extant literature, we hypothesized a priori specific involvement of amygdala, insula, periaqueductal grey (PAG) as regions of interest supporting interaction between fear processing and our experimental physiological manipulation.

\section{MATERIALS AND METHODS}

\section{Participants}

Twenty-one right-handed volunteers (12 females/9 males; mean age $=27.3$ years; $S D=2.6$; range, $23-32$ ) with no neurologic, psychiatric disorders, and other major clinical conditions, underwent detailed autonomic examination, including electrocardiogram, assessment of arterial pressure, and respiratory frequency during the Valsalva maneuver and orthostatic challenge, indicating no sign of autonomic dysfunction. The study was approved by the Santa Lucia Foundation ethical committee. Written informed consent was obtained from all participants.

\section{HRV Evaluation}

HRV analysis of normal interbeat intervals was used to index autonomic balance biased toward parasympathetic activity (Malik and Camm, 1995; Heart rate variability, 1996). The evaluation of the autonomic system consisted in the Valsalva maneuver performed consecutively three times, with a 2 -min rest, followed by a 10-min orthostatic and supine electrocardiography recording. The results obtained from 3-min recordings (extracted randomly) did not differ from the results obtained from 10-min recordings, allowing us to estimate the HRV on the basis of short time recordings. Two datasets, corresponding to three stable consecutive minutes of supine and orthostatic recordings, were analyzed using an autoregressive model (Baselli et al, 1987; Howorka et al, 2010).

Normal sinus to normal sinus (N-N) interbeat intervals were extracted to derive: (i) time domain measures (variance of $\mathrm{N}-\mathrm{N}$ intervals) and (ii) frequency domain measures (power spectral density measures indexing distributed variance of $\mathrm{N}-\mathrm{N}$ intervals as a function of frequency).

Within the time domain, we calculated the standard deviation of N-N intervals (SDNN), the square root of the mean of the squares of the differences between adjacent $\mathrm{N}-\mathrm{N}$ intervals (rMSSD), the number of differences between successive $\mathrm{N}-\mathrm{N}$ intervals greater than $50 \mathrm{~ms}$ (NN50), and the percentage of differences between adjacent $\mathrm{N}-\mathrm{N}$ intervals that are $>50 \mathrm{~ms}$ (pNN50).

The total power densities and the main power densities within high-frequency (HF) $(0.15-0.4 \mathrm{~Hz})$, low-frequency (LF) $(0.04-0.15 \mathrm{~Hz})$, and very low frequency (VLF) $(0.003-0.04 \mathrm{~Hz})$ bands were quantified in absolute values of power $\left(\mathrm{ms}^{2}\right)$.

\section{Paradigms and Procedure}

An event-related design, including two runs with 80 trials (total number of trials $=160$ ), randomly administered over $19.5 \mathrm{~min}$ of fMRI acquisition was used in this experiment (total experiment duration approximately $40 \mathrm{~min}$; Figure 1a). Within each run, 40 fearful and 40 neutral faces were randomly presented. The trials lasted on average $9700 \mathrm{~ms}$ each (range 9200-10200 ms), followed by a variable inter-trial interval, lasting on average $4 \mathrm{~s}$ (range $3050-4950 \mathrm{~ms}$ ) in $80 \%$ of the trials, and $9 \mathrm{~s}$ (range $8050-9950 \mathrm{~ms}$ ) in $20 \%$ of the trials.

During the fMRI, each participant was presented with emotional and neutral faces from the Ekman set (Ekman and Friesen, 1974). First, a fixation cross appeared for $1 \mathrm{~s}$, 


\section{${ }^{a}$ Emotional intensity paradigm}

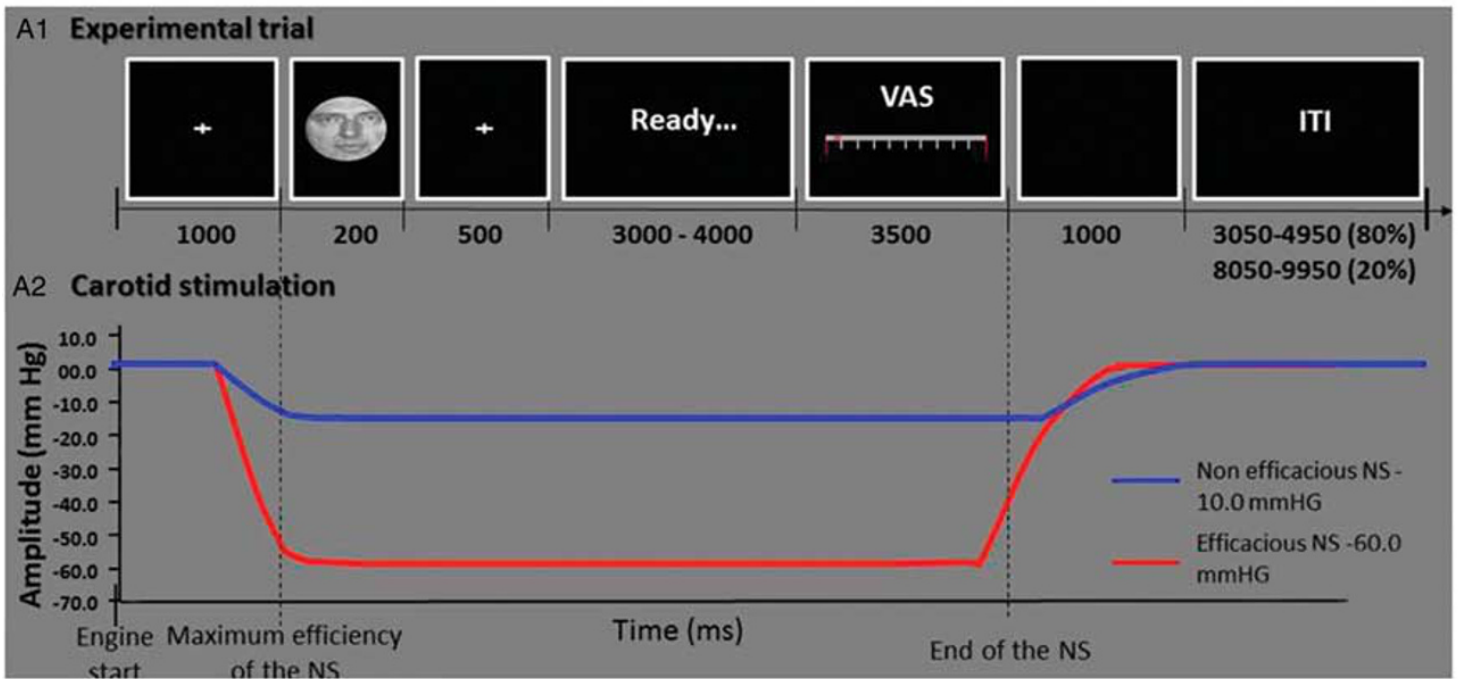

\section{b Behavioural results}

B1

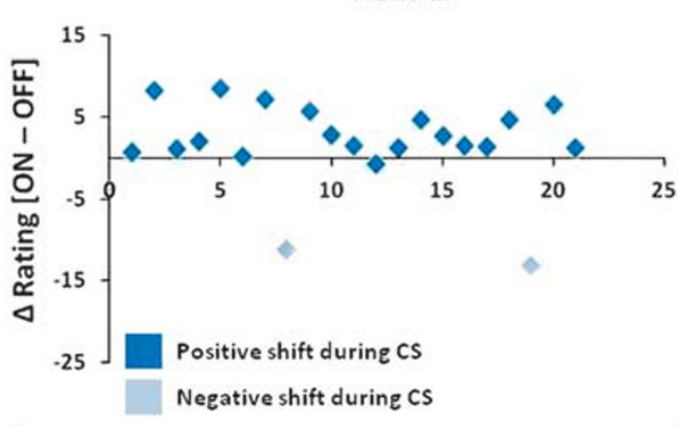

B2

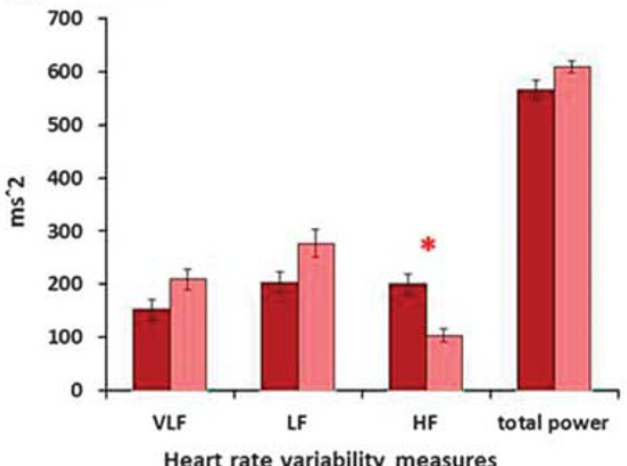

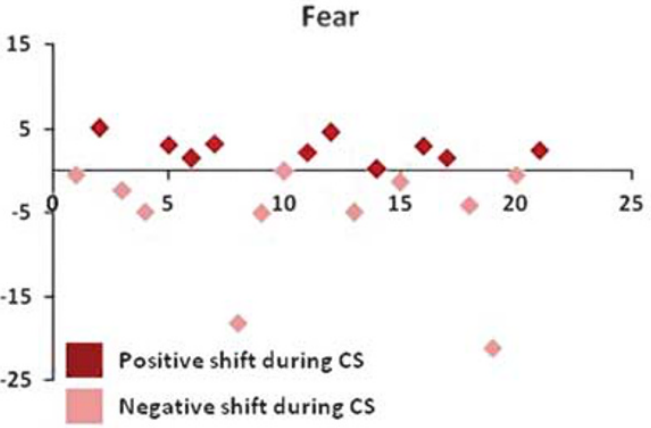

B3

- HF $y=14.19 x+196.96$
$R^{2}=0.42$

LF $y=-9.99 x+140.76$

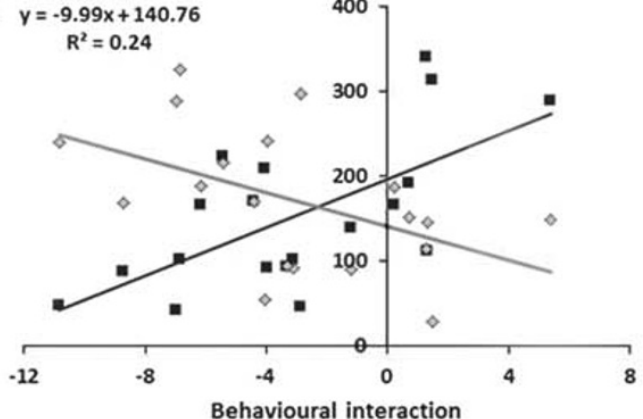

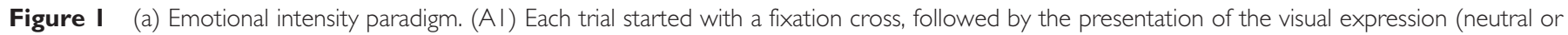

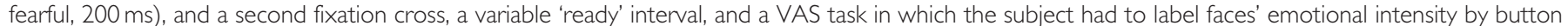
pressing on a scale ranging from 0 - no emotional intensity - to 100 - extreme emotional intensity. A variable ITI was introduced at the end of the trial. (A2) The efficacious (ON) and non-efficacious (OFF) stimulation was randomly delivered to each participant. The neck suction engine started 500 ms after the onset on the pre-face fixation cross, and reached the set value ( -10 or $-60 \mathrm{~mm} \mathrm{HG})$ at the onset of the face. Accordingly, each stimulation offsets at the end of the VAS rating event. (b) Behavioral results. (BI) Effect of CS during fearful and neutral intensity rating documented on a subject level. The $y$ axis represents $\Delta$ rating [CS ON-OFF] for neutral (left) and fearful (right) faces. Positive values indicate increased ratings during the CS ON condition. For neutral faces (left graph), the majority of participants has a positive shift during CS ON (ie, increased perceived emotional intensity). In the case of fearful

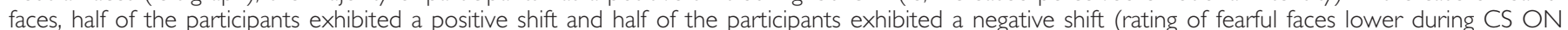
condition). (B2) Participants with a negative shift in fearful rating reported lower baseline parasympathetic activity, expressed by lower HF spectral power. (B3) HRV parameters - HF and LF-correlated with the behavioral interaction between CS and emotional rating.

followed by brief presentation of a face stimulus $(200 \mathrm{~ms}$; the $200 \mathrm{~ms}$ period was selected for because it would allow comparison with planned studies on natural baroreceptor activation within the cardiac cycle). A total of 80 fearful and 80 neutral faces were randomly presented over two runs. A post-stimulus fixation cross was then presented for $500 \mathrm{~ms}$, 
followed by a blank screen with a 'ready' message (3-4 s). A visual analogue scale (VAS) was then presented for $3500 \mathrm{~ms}$ for the participant to rate emotional intensity of the face (in this study, emotional intensity refers to the intensity of emotional experience that the facial expression evokes in the participant. In the case of neutral faces, based on previous studies, we assume that an inexpressive face is not necessarily an emotionally neutral stimulus and could, therefore, trigger an emotional experience in the individual (Phillips et al, 1997)) on a scale ranging from 0 -no emotional intensity-to 100 -extreme emotional intensity (see Figure 1). There was a variable inter-trial interval lasting on average $4000 \mathrm{~ms}$ (range $3050-4950 \mathrm{~ms}$ ) for $80 \%$ of the trials and $9000 \mathrm{~ms}$ (range $8050-9950 \mathrm{~ms}$ ) for $20 \%$ of the trials.

\section{CS Delivery}

Using a laboratory-built device for MRI, neck suction was delivered through two individual cuffs within a neck collar (Calcagnini et al, 2010; Basile et al, 2013a, b). The pressure was set by controlling the aspiration level of a vacuum source (placed in the MRI control room) by a PC. The actual pressure within each cuff of the neck collar was continuously and independently monitored. Specific placement of neck suction cuffs was tailored for each participant, using carotid angiograms acquired earlier to localize points of carotid artery bifurcation. CS was delivered in pulses of variable duration, ranging from 7200 to $8200 \mathrm{~ms}$. In order to assure an efficient influence of the ANS perturbation on the emotional elaboration of the faces, the CS was time-locked to the onset of the face stimulus and offset of the VAS. Periods of efficacious ( $-60 \mathrm{~mm} \mathrm{Hg}$ pressure) and nonefficacious $(-10 \mathrm{~mm} \mathrm{Hg}$ pressure) CS were randomly delivered. Active pulses were always followed by an intertrial interval of a variable duration (Figure 1a, panel A), during which CS was not delivered and participants were not engaged in any active task. This reduced the likelihood of baroreceptor response accommodation.

\section{Physiological Signal Acquisition and Analysis}

Electrocardiography, pulse oximetry, and respiration were recorded during fMRI (Biopac Systems Ins, CA). N-N interbeat intervals were extracted from the electrocardiography. Cardiovascular response to CS was assessed as the percentage difference between the average $\mathrm{N}-\mathrm{N}$ interval during the neck suction and the average $\mathrm{N}-\mathrm{N}$ interval during the preceding inter trial interval. No motion artifacts were detected when the CS was applied using the two-cuff device. Physiological monitoring and CS delivery did not induce an increase in radio frequency noise.

\section{fMRI Acquisition and Preprocessing}

Neuroimaging data were acquired using a head-only $3.0 \mathrm{~T}$ MR scanner (Siemens Magnetom Allegra, Siemens Medical Solutions, Erlangen, Germany). Functional brain images optimized for blood oxygenation level-dependent contrast were collected using an echo-planar $\mathrm{T} 2{ }^{*}$-weighted sequence $(\mathrm{TR}=2.08 \mathrm{~s}, 32$ axial slices, slice thickness $=2.5 \mathrm{~mm}$, gap $=1.3 \mathrm{~mm}$ ). Data were processed using MATLAB 7.0
(MathWork, Natick, MA) and SPM8 (Statistical Parametrical Mapping, http://www.fil.ion.ucl.ac.uk). In both experiments, the first four volumes were discharged to allow for T1 equilibration effects

EPI images were realigned to the first image and normalized to a standard echoplanar image template. Normalized functional scans were smoothed with a Gaussian kernel of 8-mm (full-width half maximum).

\section{Data Analysis}

A repeated measures $2 \times 2$ ANOVA was conducted to test the effect of CS on emotional processing. CS (efficacious$\mathrm{ON}$, non-efficacious-OFF) $\times$ emotion (fear, neutral) were entered as within-participant variables. A high-pass filtering to $1 / 128 \mathrm{~Hz}$ was applied to remove low-frequency noise

The stimulation-by-emotion interaction effect on ratings was then explored using post hoc t-tests Bonferronicorrected for multiple comparisons. Further, we performed a median-split analysis to explore for differences in a physiological measure in the group of participants, based on their performance on the behavioral task.

First-level analyses estimating contrasts of interest for each participant were followed by second-level mixed-effect analyses for statistical inference at the group level (Friston et al, 2002).

The first-level multiple regression model included eight conditions, four corresponding to the face event and four corresponding to the VAS task, in both cases reflecting a combination of emotion (fear, neutral) and CS (ON, OFF), which were modeled and convolved with a canonical hemodynamic response function. The four face event conditions were modeled as miniblocks, time-locked at the onset of the face with a duration of $200 \mathrm{~ms}$, whereas the four VAS conditions were time-locked at the onset of the VAS rating event with a duration of $3500 \mathrm{~ms}$. All predictors were convolved with the SPM8 hemodynamic response function, and realignment parameters were included as covariates of no interest.

At the group level, two different analyses were carried out. In a first analysis, the four conditions resulting from the emotion $\times$ CS combination (of both the face event and the VAS period) were modeled within a $2 \times 2$ within-participant ANOVA, to test for the main effect of the stimulation $(\mathrm{ON}<\mathrm{OFF}$; ON $>$ OFF) across emotional conditions, and for the interactions between the two factors [(fearON $<$ OFF) -(neutralON $<\mathrm{OFF})]$. A correlational analysis was then carried out between the difference between fearful $[\mathrm{ON}-\mathrm{OFF}]$ and neutral ratings $[\mathrm{ON}-\mathrm{OFF}]$ and brain activity during the VAS task. The statistical threshold was set to $P<0.05$, FWE corrected at the voxel level for whole brain.

Moreover, for the region-of-interest analyses, anatomical masks were constructed using the anatomical toolbox in SPM (Tzourio-Mazoyer et al, 2002) for bilateral insula. A sphere ( $6 \mathrm{~mm}$ radius) was applied on the region of the PAG, based on MNI coordinates [0 - $28-9$ ] and a $10 \mathrm{~mm}$ radius sphere on the amygdala based on coordinates [32 $\left.0 \begin{array}{ll}32 & -24\end{array}\right]$ from Garfinkel et al, (2014). Statistical threshold was set to $P<0.05-F W E$-corrected at cluster level (cluster size defined using uncorrected voxel-level threshold $P<0.005$ ).

Next, we tested whether the areas showing an effect of CS over emotional appraisal were associated with basal HRV 
measures. We considered a sphere ( $10 \mathrm{~mm}$ radius $)$ centred on peak voxel within the region-of-interest analyses described above. The association between contrast estimates from our region-of-interests and HRV measures were evaluated using Pearson correlations.

\section{RESULTS}

As expected, all subjects (but one) had an increase of R-R intervals during the efficacious stimulation (see Supplementary Figure S1 for the individual response to efficacious CS). Thus, efficacious CS engendered a significant increase in the interbeat (R-R) interval indicating autonomic perturbation (average increase in R-R interval: $19.2 \mathrm{~ms}$; pre $v s$ during CS, $t(20)=8.4, P<0.001)$. Non-efficacious CS resulted in a non-significant increase of $6.0 \mathrm{~ms}$ in (pre $v s$ during CS, $t<1)$.

\section{Behavioural Results}

We observed a significant main effect of emotion, reflecting greater intensity ratings for fear faces relative to neutral faces $(\mathrm{F}(1,18)=334.9, P<0.001)$, and no main effect of CS $(\mathrm{F}(1,20)<1)$ on intensity ratings. Importantly, we observed a significant CS-by-emotion interaction $(\mathrm{F}(1,20)=14.8$, $P<0.001)$.

For neutral faces, the efficacious CS induced a positive shift (ie, higher) emotional intensity ratings; efficacious-ON $=33.79, \mathrm{SD}=15.66$ vs non-efficacious-OFF $=32.07, \mathrm{SD}=$ 18.99). This positive shift occurred in almost all participants (Figure 1b, panel A). Planned contrasts for each emotion type revealed a trend in the effect of CS on appraisal of neutral expressions (18/20 positive events, two-tailed sign test $P<0.01)$. Across the group, there was no overall significant difference for fearful expressions (efficacious$\mathrm{ON}=66.73, \quad \mathrm{SD}=20.30 \quad v s$ non-efficacious-OFF 68.45 , $\mathrm{SD}=14.67 ; \quad t(20)=1.2, \quad P=0.1)$. However, during the appraisal of fearful stimuli, 10 participants exhibited a positive shift and 11 participants exhibited a negative shift.

We therefore performed a median-split of participants to compare HRV measures of the participants exhibiting a positive shift and those exhibiting a negative shift. Participants with a positive shift in fearful rating manifest higher basal vagal parasympathetic tone compared with those with a negative shift, expressed as higher HF spectral power and rMSSD, NN50, and pNN50 HRV timedomain indices (Figure 1b, panel B). Therefore, when controlling for the basal vagal activity of each participant, a significant difference emerged for fear ratings when comparing ON vs OFF conditions $(66.73$ vs 68.46, $\mathrm{F}(1,19)=$ 4.96, $P<0.05)$.

\section{Relation to HRV Parameters}

HRV parameters correlated with the magnitude of the behavioral expression of the stimulation-by-emotion interaction on intensity ratings ([fearON-OFF] - [neutralON$\mathrm{OFF}]$ ) (Figure 1b, panel $\mathrm{C}$ ). A positive correlation was evident between the behavioral interaction and time domain measures of parasympathetic response: NN50 $(r=0.48$, $P<0.02)$, pNN50 $(r=0.50, P<0.03)$. Similarly, this behavioral interaction negatively correlated with LF $(r=-0.49$,
$P<0.03)$ and VLF spectrum $(r=-.55, P<0.01)$, yet positively correlated with HF spectrum $(r=-.65$, $P<0.02)$. In all the cases, the interaction was driven by a correlation with the simple effect of CS on perceived intensity of fear stimuli (fear ON-OFF; pNN50 $r=0.49$; HF $r=0.48$, VLF $r=-0.57$, LF $r=-0.47$; all $P<0.05$ ), whereas no correlation was evident with the effect of CS on neutral ratings (neutral $\mathrm{ON}-\mathrm{OFF}$ ). This result extends the findings of the median-split analysis to indicate that the individual basal vagal activity differently affected the modulation of emotional ratings by CS: Individuals with higher basal HRV exhibited a positive shift in the rating of fearful faces during the ON condition. Notably, the same influence was not evident during the neutral rating condition. In order to examine whether the carotid effect was confined among participants with high or low HRV, a second median-split control analysis was conducted, splitting the group in 'high' and 'low' HRV participants. The difference between $\mathrm{ON}$ and $\mathrm{OFF}$ in rating of fearful expressions was coherent with the first median split analysis, that is, the group of 'high HRV' group reported a positive shift in fearful rating during CS ON, whereas the 'low HRV' group reported a negative shift, although both effects were not significant $(P<0.1$ one-tailed $t$ test) (see Supplementary Material for a graphic representation, Supplementary Figure S2).

\section{Neuroimaging Results}

Main effect of efficacious CS. A whole-brain neuroimaging analysis was conducted to test the effect of CS during the appraisal of facial expressions (ie, VAS rating period), although an exploratory analysis has also been conducted to test the effect of CS on perception of facial expressions (ie, face presentation event, see Figure 1).

Consistent with our knowledge concerning the delay in effects of maximum baroreceptor stimulation with this method from our previous studies (eg, Basile et al, 2013a, b), we anticipated effects to emerge after the face stimuli were presented. The analysis focused on the VAS event revealed a significant main effect of CS during the rating of face stimuli, irrespective of emotion for stimulation $\mathrm{ON}<\mathrm{OFF}$ (see Table 1) manifest as a distributed decrease in brain activity during efficacious stimulation in the following areas: bilateral hippocampus, bilateral amygdala, left thalamus, and temporal fusiform areas (see Figure 2 and Table 1 for the complete list of brain areas). The region-ofinterest analysis revealed a significant effect also in bilateral insula. No positive effects of efficacious CS on brain activity (ON > OFF) were evident.

Regarding face perception, the results are marginal for the purpose of the current study, and are presented as Supplementary Material. Overall, a significant main effect was evident during face perception, this manifested both as increased neural activity in the lateral occipital cortex bilaterally and decreased activity in bilateral temporal pole (see Supplementary Figure S3 and Supplementary Table S1). Moreover, increased activity was present during the perception of fearful over neutral faces irrespective of CS, in left occipital fusiform gyrus, left anterior cingulated gyrus, and right postcentral gyrus. 
Table I Brain Activation Underlying Main Effect of Carotid Stimulation, Collapsed Over Specific Emotion Type

\begin{tabular}{|c|c|c|c|c|}
\hline \multirow[t]{2}{*}{ Brain region } & \multicolumn{2}{|c|}{ Cluster } & \multicolumn{2}{|r|}{ Voxel } \\
\hline & k & P FWE & $\mathbf{Z}$ & MNI xyz \\
\hline Temporal pole $\mathrm{R}$ & 31860 & $0.000^{\mathrm{a}}$ & 5.63 & $3414-40$ \\
\hline Temporal pole L & & & 5.19 & $-42 \mid 4-32$ \\
\hline Superior frontal gyrus $\mathrm{R}$ & & & 5.09 & 222052 \\
\hline Superior frontal gyrus $L$ & & & 4.89 & -202248 \\
\hline Hippocampus L & & & 5.08 & $-24-12-20$ \\
\hline Hippocampus R & & & 4.78 & $26-10-22$ \\
\hline Postcentral gyrus/precuneus R & & & 5.02 & $6-3658$ \\
\hline Postcentral gyrus L & & & 4.44 & $-44-1228$ \\
\hline Parahippocampal gyrus R & & & 4.79 & $36-32-14$ \\
\hline Amygdala $\mathrm{R}$ & & & 4.78 & $264-24$ \\
\hline Amygdala L & & & 4.65 & $-28-4-24$ \\
\hline Middle temporal gyrus $\mathrm{R}$ & & & 4.72 & $52-2-24$ \\
\hline Temporal fusiform area $L$ & & & 4.54 & $-34-40-12$ \\
\hline $\begin{array}{l}\text { Superior parietal lobule/ } \\
\text { postcentral gyrus R }\end{array}$ & & & 4.54 & $32-3846$ \\
\hline Thalamus L & & & 4.52 & $-16-2218$ \\
\hline Cerebellum, L VI & 561 & $0.009^{\mathrm{a}}$ & 3.84 & $-8-64-18$ \\
\hline Insula $L$ & 9 & $0.02^{\mathrm{b}, \mathrm{c}}$ & 3.56 & $-32-26 \mid 6$ \\
\hline Insula R & 12 & $0.02^{\mathrm{b}, \mathrm{c}}$ & 3.41 & $44-6-18$ \\
\hline Brainstem, midbrain & 231 & $0.00 I^{b}$ & 4.33 & $-2-16-18$ \\
\hline
\end{tabular}

${ }^{\mathrm{a}} \mathrm{FWE}$ whole brain cluster level.

${ }^{\mathrm{b}} \mathrm{FWE}$ ROI.

${ }^{\mathrm{c}}$ FWE whole brain peak level.

Interaction between CS and emotional rating condition. We next tested for interaction between CS (ON, OFF) and emotion ([fearON $<\mathrm{OFF}$ ] $v s$ [neutral $\mathrm{ON}>\mathrm{OFF}]$ ) to investigate the specific effect of CS on emotional appraisal. Conversely, the activity within left amygdala showed a significant CS-by-emotion interaction $(T=2.88,19$ voxel, $P<0.05$ FWE-corrected for small-volume, Figure $2 \mathrm{~b}$, panel A) during the appraisal of facial expressions (ie, the VAS period). Analysis of the mean activation from left amygdala showed that the interaction $(\mathrm{F}(1,20)=7.42, P<0.01)$ was particularly driven by attenuation of amygdala reactivity during CS when appraising the intensity of fear stimuli (fearON $v$ s fearOFF $t(20)=4.52, P<0.001$ ) as no difference was evident when comparing neutralON $v s$ neutralOFF activation in left amygdala $(t(20)<1)$ (Figure $2 \mathrm{~b}$, panel B).

Consistent with our expectations, we did not observe any significant CS-by-emotion interaction during the perception of faces.

Correlations with emotional ratings. We tested for regions whose activity correlated directly with the behavioural shift induced by CS during fearful and neutral rating. Overall, a positive correlation was evident between changes in the rating of fearful faces [fearful $\mathrm{ON}-\mathrm{OFF}$ ] and the relative attenuation of brain activation by CS. A correlation was evident within bilateral thalamus, hippocampus, cerebellum, middle temporal gyrus, putamen and globus pallidus, frontal orbital cortex, bilateral amygdala and bilateral insula, and PAG (see Figure 3a and Table 2 for the complete list of brain areas).

We extracted the mean activation from amygdala, insula, and PAG in order to perform additional analyses. A significant correlation was obtained between HRV parameters and amygdala activation during fear rating. Basal LF, $\mathrm{HF}$, and RMSSD parameters correlated with activity in left amygdala (LF $r=-0.55, P<0.015$; HF $r=0.47, P<0.05$; RMSSD $r=0.45, P=0.05)$ and PAG (LF $r=-0.48, P<0.05$; HF $r=0.52, P<0.05$, Figure 2a). Analysis of the mean activation from left amygdala showed that the individuals who had a negative shift in the rating of fearful faces reported a stronger attenuation in the activity of amygdala and lower basal vagal activity (Figure $3 \mathrm{~b}$ ).

With neutral faces, a negative correlation was evident between the change in neutral rating $[\mathrm{ON}-\mathrm{OFF}]$ and in the attenuated activation of PAG during active CS $(T=3.46,7$ voxel, $\quad P<0.037$ FWE-corrected for small-volume, Figure $3 c)$.

\section{DISCUSSION}

The aim of this study was to explore how afferent cardiovascular information contributes to the processing of emotion stimuli, notably the fear signals of others. Using stimulation of carotid baroreceptors to perturb parasympathetic drive, we tested for hypothesized effects on the neural processing and appraisal of fearful (relative to neutral) facial expressions. In addition, we explored how individual differences in autonomic state (particularly the basal vagal parasympathetic tone) predicted the influence of CS on emotional processing.

Regarding the effect of CS during the appraisal of facial emotions, the observed brain activity is consistent with earlier observations that activation of arterial baroreceptors may inhibit sensory processing and cortical excitability (Koriath et al, 1987).

Here, efficacious CS resulted in attenuated activation across cortical and subcortical brain areas during the facial appraisal, including insula, amygdala, hippocampus, thalamus, and brainstem. Many of these brain centers are implicated in autonomic regulation (Critchley, 2005a; Kimmerly et al, 2005). In a previous study, our group used the same methodology to describe the modulation by CS of activity within regions, including insula, amygdala, and PAG, both at rest and when participants were engaged in a cognitive task (Basile et al, 2013a), or during processing of sad, happy, angry, and neutral faces (Basile et al, 2013b).

Conversely, the effect of the CS during early events of the trial (ie, face presentation) affected mainly occipital and temporal areas, consistent with the expected delay in effects of maximum baroreceptor stimulation with this method on our areas of interest (ie, insula, amygdaala, PAG).

A noteworthy aspect of the present study is that CS elicited a differential effect on neural activity during the appraisal of fearful and neutral faces. Activity within amygdala was affected by CS exclusively during the 


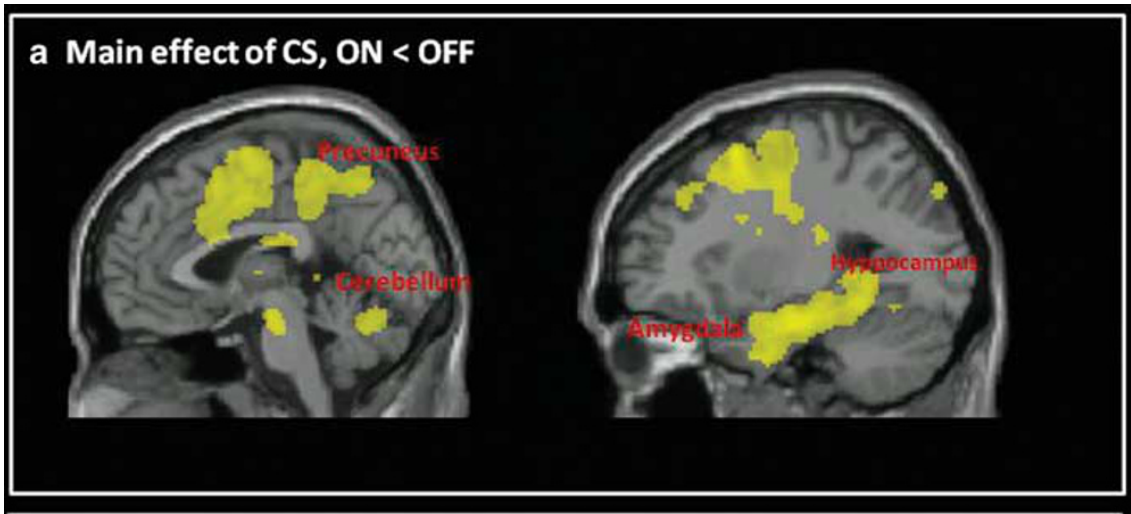

\section{b Interaction [fear ON < fear OFF] vs. [neutral ON > neutral OFF]}

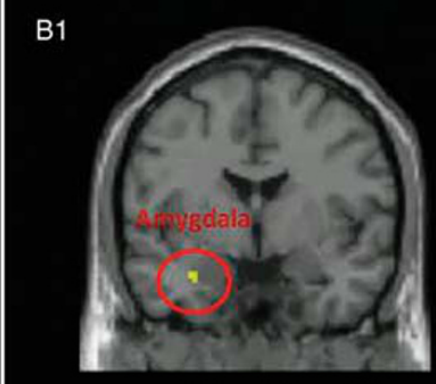

$x=-30 y=0 z=-25$
B2

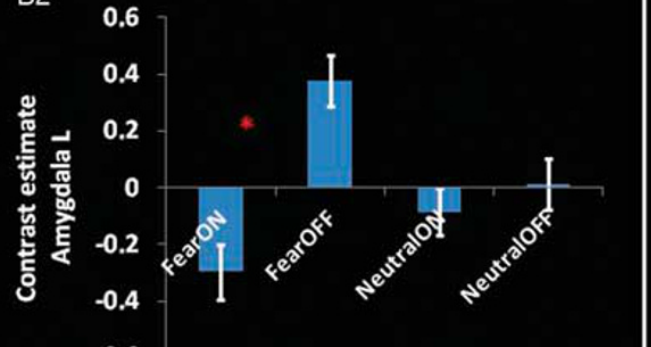

$-0.6$

Figure 2 (a) Main effect of CS (averaged across emotional condition). Notably, brain activity was attenuated during CS in bilateral hippocampus, amygdala, and insula (see Table I for the complete list of brain areas). (b) (BI) An effect of the CS $\times$ emotional condition interaction emerged in the activity of left amygdala. (B2) The interaction was mainly driven by the attenuation of activity in left amygdala during the rating of fearful faces.

appraisal of fearful faces. In addition, the attenuation of left amygdala activity during the appraisal of fearful expressions predicted the behavioral consequences, as reflected in the magnitude of the behavioral interaction. Thus, our results extend mechanistic understanding of the role of the amygdala in the perception and processing of threat, by highlighting the integration of viscerosensory signaling and autonomic control with the neural and behavioral sensitivity to the fear signals of other people (Zald, 2003; Phelps and LeDoux, 2005; Garfinkel et al, 2014).

These insights were further endorsed by the observation that at the level of individual differences, with individuals with low basal parasympathetic activity (across HRV-related measures) manifesting a decreased perception of fear during the CS. The degree of change in the behavioral ratings evoked by CS correlated with activity across a set of related regions including insula, amygdala, hippocampus, and orbitofrontal cortex. We were particularly interested in the putative role of vagal parasympathetic tone as an index of adaptive emotional regulatory capacity (Friedman, 2007). Low vagal activity is associated with anticipatory anxiety and hypervigilance toward potential threat (Thayer et al, 2009), and interestingly those individuals who exhibited a negative shift in fearful ratings during the CS manifest the greatest attenuation of the amygdala activity and had lower parasympathetic tone. We showed this effect also in a direct correlation between HRV measures and attenuation of amygdala and PAG activity when appraising fear faces. These findings strongly endorse the perspective that the degree to which viscerosensory afferent information impacts upon both neural reactivity and emotional processing is highly dependent on the integrity of parasympathetic autonomic control and individual characteristics in basal vagal cardiovascular regulation. Lower vagal activity has been linked to reduced prefrontal inhibitory control over the amygdala (Thayer et al, 2009), and is observed in patients with generalized anxiety disorders, panic disorder, and even children of patients with panic disorder (see Friedman, 2007 for a review; Srinivasan et al, 2002). Here, we show that individuals with lower HRV were more responsive towards the CS, by reporting a stronger deactivation of amygdala and lower rating of fearful stimuli, according to the literature reporting that higher parasympathetic tone is associated with reduced sensitivity to fear perception (Thayer et al, 2009).

Individuals with higher HRV were, conversely, less responsive to CS, showing a less evident effect of the CS on amygdala and a weak positive shift in fearful rating. This raises the possibility that the relationship between parasympathetic tone and fear may not be linear (but instead follows an inverted U-shaped function), or implicates additional mechanisms such as enhanced interoceptive accuracy at lower heart rates (Pollatos et al, 2007). Future 


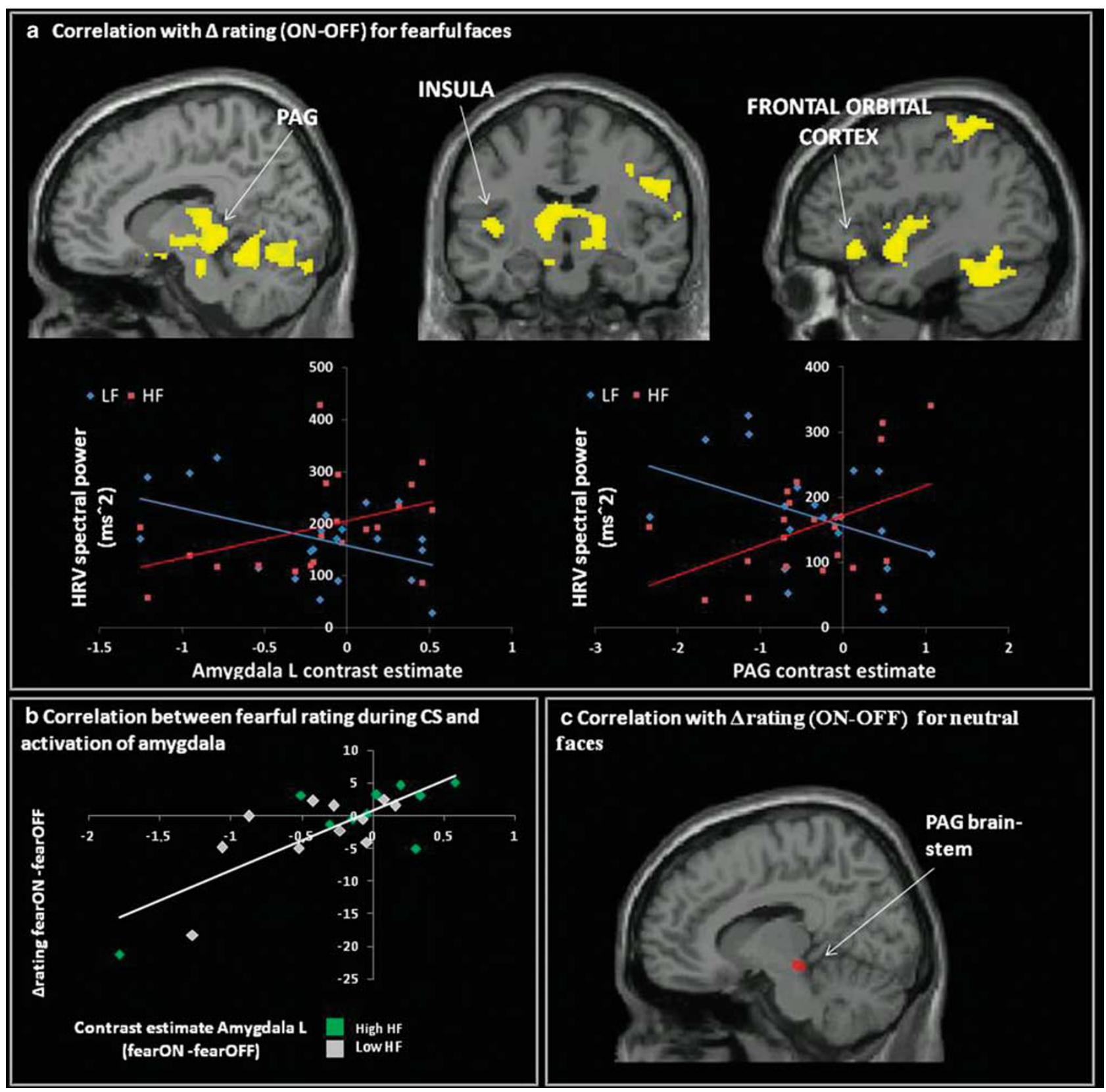

Figure 3 (a) Graph reporting brain areas significantly correlating with the behavioral shift in fearful rating during CS efficacious stimulation. The activation in left amygdala and PAG correlated with HRV measures, with individuals with higher LF and lower HF indexes reporting stronger attenuation during CS efficacious stimulation. (b) The correlation between the shift in fearful rating and the neural activation in left amygdala during the appraisal of fearful faces, in individuals having higher and lower HF. Overall, Individuals exhibiting a negative shift in fearful rating had the strongest deactivation of the amygdala and lower parasympathetic tone. (c) The behavioral shift in neutral rating during CS efficacious stimulation correlated with the activity in PAG area.

research is needed to fully delineate the mechanisms underlying the complex interplay between the basal HRV, stimulation of the parasympathetic tone, and the appraisal of emotions.

The influence of baroreceptor afferents on the processing of fearful stimuli was recently investigated by Garfinkel et al, (2014). Here, timing brief fear stimuli to systole, during natural baroreceptor firing, is associated with enhanced processing of fear and corresponding increased neural activity in regions including amygdala. There is moreover an attenuation of the processing of fearful stimuli during diastole. This latter observation may account for the apparent paradoxical findings with present study, where attenuated fear processing (albeit over longer time periods) occurred with the lengthening of inter-beat interval following artificial baroreceptor stimulation. Prolongation of diastole during CS may partly account for these apparently mixed findings, but there remains more to understand about the timing of baroreceptor influences on emotion and perception. Nevertheless, both studies highlight an important viscerosensory cardiovascular influence, specifically linked to the baroreflex, on the processing of threat signals and fear stimuli. Here, we quantify the neural and behavioral impact of baroreceptor 
Table 2 Brain Areas That Positively Correlated With a Rating (On-Off) of Fearful Faces

\section{Correlation with fear rating}

\begin{tabular}{|c|c|c|c|c|}
\hline \multirow[t]{2}{*}{ Brain region } & \multicolumn{2}{|c|}{ Cluster } & \multicolumn{2}{|r|}{ Voxel } \\
\hline & $\mathbf{k}$ & P FWE & $\mathbf{Z}$ & MNI xyz \\
\hline Thalamus R & 9182 & $0.00^{\mathrm{a}}$ & 4.89 & $22-34-2$ \\
\hline Thalamus L & & & 4.02 & $-10-1812$ \\
\hline Temporal occipital fusiform $\mathrm{R}$ & & & 4.64 & $22-52-16$ \\
\hline Temporal occipital fusiform $L$ & & & 3.89 & $-36-56-14$ \\
\hline Hippocampus L & & & 4.51 & $-24-24-6$ \\
\hline Putamen & & & 4.21 & $-32-48$ \\
\hline Cerebellum & & & 4.07 & $-38-50-30$ \\
\hline Middle temporal pole & & & 3.99 & $-566-26$ \\
\hline Parahippocampal gyrus, posterior $\mathrm{L}$ & & & 3.84 & $-28-36-16$ \\
\hline Globus pallidum $L$ & & & 3.75 & $-14-4-4$ \\
\hline Inferior temporal gyrus $L$ & & & 3.72 & $56-56-14$ \\
\hline Frontal orbital cortex & & & 3.45 & $-3830-12$ \\
\hline Brain-stem & & & 3.42 & $10-20-22$ \\
\hline Postcentral gyrus $L$ & 867 & $0.03^{\mathrm{a}}$ & 4.48 & $-44-3860$ \\
\hline Superior parietal lobule & & & 2.69 & $-22-4656$ \\
\hline Precentral gyrus $\mathrm{R}$ & 718 & $0.06^{\mathrm{a}}$ & 4.19 & $46-432$ \\
\hline Amygdala $L$ & 39 & $0.003^{b, c}$ & 3.92 & $-364-16$ \\
\hline Insula L & 24 & $0.02^{\mathrm{b}, \mathrm{c}}$ & 3.47 & $-44-126$ \\
\hline Insula R & 12 & $0.01^{b, c}$ & 3.62 & $32-26-12$ \\
\hline PAG & $17 \mid$ & $0.01^{b, c}$ & 3.37 & $12-30-6$ \\
\hline
\end{tabular}

${ }^{\mathrm{b} F W E}$ whole brain peak level.

aFWE whole brain cluster level.

${ }^{\mathrm{C}} \mathrm{FWE}$ ROI.

firing on fear processing and extend previous results by noting the dependence of this effect on basal autonomic characteristics of individuals.

Overall, CS showed less pronounced effects on the ratings of neutral faces. PAG activity correlated with stimulationinduced change in the rating of neutral faces. The PAG region is previously implicated in the coupling of peripheral arousal state to emotional processing and expression of fear (Mobbs et al, 2009; Gray et al, 2012; Linnman et al, 2012). Consistently with these previous studies, our data show that a modulation of the neural activity in the PAG area caused by CS results in an increased evaluation of the emotional intensity in neutral faces.

Taken together, our results demonstrate how viscerosensory and autonomic perturbation affect appraisal of fearful and neutral faces at neural and subjective levels. Our study highlights the utility of integrating physiological and neuroimaging techniques to gain detailed insight into mechanisms underlying influential theories of emotion. Our device stimulated the carotid sinus baroreceptors and increased the heartbeat interval, affecting activation across distributed brain areas. Through the use of CS, we directly evaluated the influence of peripheral bodily arousal on emotional appraisal and further demonstrate the influence of individual basal vagal tone on these affective processes.
The current study not only provides neurophysiological insights on the interaction between body and brain, but can also potentially contribute to the understanding of pathophysiological conditions, not least the expression and maintenance of anxiety disorders (Garfinkel et al, 2014), and suggests that treatments for anxiety might target baroreflex-related central pathways.

\section{FUNDING AND DISCLOSURE}

The authors declare no conflict of interest.

\section{ACKNOWLEDGEMENTS}

E. Makovac is supported by the grant RF09.150.1. HDC and SNG Aare supported by the ERC via and advanced grant ERC-2012-ADG_20120411 and by the Dr Mortimer and Dame Theresa Sackler Foundation via the Sackler Centre of Consciousness Science, University of Sussex

\section{REFERENCES}

Barrett LF, Lindquist K, Gendron M (2007). Language as context for the perception of emotion. Trends Cogn Sci 11: 327-332.

Baselli G, Cerutti S, Civardi S, Lombardi F, Malliani A, Merri M et al (1987). Heart rate variability signal processing: a quantitative approach as an aid to diagnosis in cardiovascular pathologies. Int J Biomed Comput 20: 51-70.

Basile B, Bassi A, Calcagnini G, Caltagirone C, Bozzali M (2013b). Effect of parasympathetic stimulation on brain activity during emotional processing. Proceedings of the Human Brain Mapping annual meeting June 8-12, 2014, Hamburg, Germany.

Basile B, Bassi A, Calcagnini G, Strano S, Caltagirone C, Macaluso E et al (2013a). Direct stimulation of the autonomic nervous system modulates activity of the brain at rest and when engaged in a cognitive task. Hum Brain Mapp 34: 1605-1614.

Calcagnini G, Mattei E, Triventi M, Basile B, Bassi A, Bozzali M et al (2010). Investigation of the autonomic nervous system control of cardiovascular variables using fMRI and carotid stimulation. Computing in Cardiology 37: 529-532.

Cannon WB (1927). The James-Lange theory of emotions. Am J Psychol 39: 115-1124.

Cooper VL, Hainsworth R (2009). Carotid baroreflex testing using the neck collar device. Clin Auton Res 19: 102-112.

Craig AD (2002). How do you feel? Interoception: The sense of the physiological condition of the body. Nat Rev Neurosci 3: 655-666.

Critchley HD (2005a). Neural mechanisms of autonomic, affective, and cognitive integration. J Comp Neurol 493: 154-166.

Critchley HD, Mathias CJ, Dolan RJ (2002). Fear conditioning in humans: the influence of awareness and autonomic arousal on functional neuroanatomy. Neuron 33: 653-663.

Critchley HD, Rotshtein P, Nagai Y, O’Doherty J, Mathias CJ, Dolan RJ (2005b). Activity in the human brain predicting differential heart rate responses to emotional facial expressions. Neuroimage 24: 751-762.

Critchley HD, Wiens S, Rotshstein P, Öhman A, Dolan RJ (2004). Neural systems supporting awareness. Nat Neurosci 7: 189-195.

Damasio AR (1994). Descartes' Error: Emotion, Reason and the Human Brain. Grosset/Putnam: New York.

Dolan RJ (2002). Emotion, cognition, and behavior. Science 298: 1191-1194.

Edwards L, Inui K, Ring C, Wang X, Kakigi R (2008). Pain-related evoked potentials are modulated across the cardiac cycle. Pain 137: $488-494$. 
Ekman P, Friesen WV (1974). Detecting deception from body or face. J Pers Soc Psychol 29: 288-298.

Ekman P, Levenson RW, Friesen WV (1983). Autonomic nervous system activity distinguishes among emotions. Science 221: 1208-1210.

Fadel PJ, Ogoh S, Keller DM, Raven PB (2003). Recent insights into carotid baroreflex function in humans using the variable pressure neck chamber. Exp Physiol 88: 671-680.

Friedman BH (2007). An autonomic flexibility-neurovisceral integration model of anxiety and cardiac vagal tone. Biol Psychol 74: 185-199.

Friston KJ, Glaser DE, Henson RN, Kiebel S, Phillips C, Ashburner J (2002). Classical and Bayesian inference in neuroimaging: applications. Neuroimage 16: 484-512.

Garfinkel SN, Barrett AB, Minati L, Dolan RJ, Seth AK, Critchley HD (2013). What the heart forgets: Cardiac timing influences memory for words and is modulated by metacognition and interoceptive sensitivity. Psychophysiology 50: 505-512.

Garfinkel SN, Minati L, Gray MA, Seth AK, Dolan RJ, Critchley HD (2014). Fear from the heart: sensitivity to fear stimuli depends on individual heartbeats. J Neurosci 34: 6573-6582.

Gianaros PJ, Onyewuenyi IC, Sheu LK, Christie IC, Critchley HD (2012). Brain systems for baroreflex suppression during stress in humans. Hum Brain Mapp 33: 1700-1716.

Gray MA, Beacher FD, Minati L, Nagai Y, Kemp AH, Harrison NA et al (2012). Emotional appraisal is influenced by cardiac afferent information. Emotion 12: 180-191.

Hamann S, Mao H (2002). Positive and negative emotional verbal stimuli elicit activity in the left amygdala. NeuroReport 13: 15-19.

Harrison N, Singer T, Rotshtein P, Dolan RJ, Critchley HD (2006). Pupil size modulates the empathic experience of sadness. Soc Cogn Affect Neurosci 1: 1-13.

Harrison NA, Brydon L, Walker C, Gray MA, Steptoe A, Critchley HD (2009). Inflammation causes mood change through alterations in subgenual cingulate activity and mesolimbic connectivity. Biol Psychiatry 66: 407-414.

Heart rate variability. Standards of measurement, physiological interpretation, and clinical use (1996). Task Force of the European Society of Cardiology and the North American Society of Pacing and Electrophysiology. Eur Heart J 17: 354-381.

Howorka K, Pumprla J, Jirkovska A, Lacigova S, Nolan J (2010). Modified orthostatic load for spectral analysis of shortterm heart rate variability improves the sensitivity of autonomic dysfunction assessment. J Diabetes Complications 24: 8-54.

Jacobs BL, Abercrombie ED, Fornal CA, Levine ES, Morilak DA, Stafford IL (1991). Single-unit and physiological analyses of brain norepinephrine function in behaving animals. Prog Brain Res 88: 159-165.

James W (1894). Physical basis of emotion. Psychol Rev 1: 516-529 (reprinted in 1994. Psychological Rev 101: 205-210).

Kimmerly Ds,, O'Leary DD, Menon RS, Gati JS, Shoemaker JK (2005). Cortical regions associated with autonomic cardiovascular regulation during lower body negative pressure in humans. J Physiol 569: 331-345.

Koriath JJ, Lindholm E, Landers DM (1987). Cardiac-related cortical activity during variations in mean heart rate. Int $J$ Psychophysiol 5: 289-299.

LeDoux JE (2000). Emotion circuits in the brain. Annu Rev Neurosci 23: 155-184.
Linnman C, Moulton EA, Barmettler G, Becerra L, Borsook D. (2012). Neuroimaging of the periaqueductal gray: state of the field. Neuroimage 60: 505-522.

Malik M, Camm AJ (1995). Heart Rate Variability. Futura Armonk, NY, pp 393-406.

Mobbs D, Marchant JL, Hassabis D, Seymour B, Tan G, Gray MA et al (2009). From threat to fear: The neural organization of defensive fear systems in humans. J Neurosci 29: 12236-12243.

Morilak DA, Fornal C, Jacobs BL (1986). Single unit activity of noradrenergic neurons in locus coeruleus and serotonergic neurons in the nucleus raphe dorsalis of freely moving cats in relation to the cardiac cycle. Brain Res 399: 262-270.

Phan KL, Taylor SF, Welsh RC, Ho SH, Britton JC, Liberzon I (2004). Neural correlates of individual ratings of emotional salience: a trial-related fMRI study. NeuroImage 21: 768-780.

Phelps EA, LeDoux JE (2005). Contributions of the amygdala to emotion processing: from animal models to human behavior. Neuron 48: 175-187.

Phillips ML, Young AW, Senior C, Brammer M, Andrew C, Calder AJ et al (1997). A specific neural substrate for perceiving facial expressions of disgust. Nature 389: 495-498.

Pollatos O, Herbert BM, Matthias E, Schandry R (2007). Heart rate response after emotional picture presentation is modulated by interoceptive awareness. Int J Psychophysiol 63: 117-124.

Quintana DS, Guastella AJ, Outhred T, Hickie IB, Kemp AH (2012). Heart rate variability is associated with emotion recognition: direct evidence for a relationship between the autonomic nervous system and social cognition. Int J Psychophysiol 86: 168-172.

Sagawa K (1983). Baroreflex control of systemic arterial pressure and vascular bed. In Shepard JT, Abboud FM, Geiger SR (eds). Handbook of Physiology, The Cardiovascular System: Peripheral Circulation and Organ Blood Flow. American Physiological Society: Bethesda, MD, pp 453-496.

Schachter S, Singer JE (1962). Cognitive, social and physiological determinants of emotional state. Psychol Rev 69: 379-399.

Spielberger CD, Gorsuch RL, Lushene R, Vagg PR, Jacobs GA (1983). Manual for the State-Trait Anxiety Inventory. Consulting Psychologists Press: Palo Alto, CA.

Srinivasan K, Ashok MV, Vaz M, Yeragani VK (2002). Decreased chaos of heart rate time series in children of patients with panic disorder. Depress Anxiety 15: 159-167.

Thayer J, Brosschot J (2005). Psychosomatics and psychopathology: looking up and down from the brain. Psychoneuroendocrinology 30: 1050-1058.

Thayer JF, Hansen AL, Saus-Rose E, Johnsen BH (2009). Heart rate variability, prefrontal neural function and cognitive performance: The neurovisceral integration perspective on selfregulation, adaptation, and health. Ann Behav Med 37: 141-153.

Thayer JF, Lane RD (2000). A model of neurovisceral integration in emotion regulation and dysregulation. J Affect Disord 61: 201-216.

Tzourio-Mazoyer N, Landeau B, Papathanassiou D, Crivello F, Etard O, Delcroix N et al (2002). Automated anatomical labeling of activations in SPM using a macroscopic anatomical parcellation of the MNI MRI single-subject brain. Neuroimage 15: 273-289.

Wilkinson M, McIntyre D, Edwards L (2013). Electrocutaneous pain thresholds are higher during systole than diastole. Biol Psychol 94: 71-73.

Zald DH (2003). The human amygdala and the emotional evaluation of sensory stimuli. Brain Res Brain Res Rev 41: 88-123.

Supplementary Information accompanies the paper on the Neuropsychopharmacology website (http://www.nature.com/npp) 